\title{
ON DIVISION SUBRINGS NORMALIZED BY ALMOST SUBNORMAL SUBGROUPS IN DIVISION RINGS
}

\author{
TRINH THANH DEO, MAI HOANG BIEN, AND BUI XUAN HAI
}

\begin{abstract}
Let $D$ be a division ring with infinite center, $K$ a proper division subring of $D$ and $N$ an almost subnormal subgroup of the multiplicative group $D^{*}$ of $D$. The aim of this paper is to show that if $K$ is $N$-invariant and $N$ is non-central, then $K$ is central. Some examples of almost subnormal subgroups in division rings that are not subnormal are also given.
\end{abstract}

\section{INTRODUCTION}

Let $G$ be a group. Recall that a subgroup $N$ of $G$ is said to be almost subnormal in $G$ if there exists a series of subgroups

$$
N=N_{r} \leq N_{r-1} \leq \ldots \leq N_{1} \leq N_{0}=G
$$

such that for any $0 \leq i<r, N_{i+1}$ is normal in $N_{i}$ or $\left[N_{i}: N_{i+1}\right]$ is finite. Such a series is called an almost normal series of $N$ in $G$ (see [6]). We say that $N$ is an almost $r$-subnormal subgroup (or $N$ is an almost subnormal subgroup of depth $r$ ) if $r$ is the smallest number among all almost normal series (1) of $N$ in $G$. By definition, every subnormal subgroup in a group is almost subnormal. The class of almost subnormal subgroups of skew linear groups was first mentioned in [18]. Recently, it was shown that if $D$ is a division ring with infinite center and if $n>1$, then every almost subnormal subgroup of $\mathrm{GL}_{n}(D)$ is normal [12, Theorem 3.3]. However, the case of degree 1 , that is, the group $D^{*}$, is totally different. The construction by Hazrat and Wadsworth in [7, Example 8] of non-normal maximal subgroups of finite index in certain division rings shows that there exist many division rings whose multiplicative groups contain almost 1-subnormal subgroups which are not subnormal. In Section 2, we will show that for every positive integer $r$, there exists a division ring whose multiplicative group contains an almost $r$-subnormal subgroup which is not subnormal.

Let $D$ be a division ring with center $F$ and $K$ a division subring of $D$. Assume that $N$ is a non-central subgroup of $D^{*}$ such that $K$ is normalized by $N$, that is, $x K x^{-1}=K$ for any $x \in N$. Then, we say that $K$ is $N$-invariant. The well-known Cartan-Brauer-Hua Theorem states that if $K^{*}$ is normal in $D^{*}$, that is, $K$ is $D^{*}$ invariant, then $K \subseteq F$ or $K=D$ [10, p. 211, 13.17]. Herstein, Scott and Stuth then proved that if $N$ is a subnormal subgroup of $D^{*}$, then $K \subseteq F$ or $K=D$

Key words and phrases. division ring; almost subnormal subgroup; generalized group identity. 2010 Mathematics Subject Classification. 16K20, 16K40, 16R50.

This work is funded by Vietnam National Foundation for Science and Technology Development (NAFOSTED) under Grant No. 101.04-2016.18. 
(see [8, [14, p. 439] and [16]). This result is sometimes referred to as the CartanBrauer-Hua Theorem for subnormal subgroups in division rings. There is a vast number of applications on studying subgroups in division rings.

In this note, we will extend these results for the case when $N$ is an almost subnormal subgroup of $D^{*}$ and $F$ is infinite. We will prove that if $K$ is a division subring of $D$ such that $K$ is $N$-invariant, then $K \subseteq F$ or $K=D$.

Our notation in this paper is standard. In particular, the symbol $[x, y]$ is denoted for $x y x^{-1} y^{-1}$.

\section{EXAMPles OF ALMOST SUBNORMAL SUBGROUPS IN DIVISION RINGS THAT ARE NOT SUBNORMAL}

In this section, for every positive integer $r$, we show the existence of a division ring $D$ containing an almost $r$-subnormal subgroups $N$ of $D^{*}$. Our example depends on Mal'cev-Neumann division rings of free groups over a base division ring, so we first recall some necessary results on this class of division rings.

Let $G$ be a group. For an element $x \in G$, we construct a sequence of normal subgroups

$$
G=\langle x\rangle_{0} \triangleright\langle x\rangle_{1} \triangleright\langle x\rangle_{2} \triangleright \ldots \triangleright\langle x\rangle_{n} \triangleright \ldots
$$

of $G$ containing $x$ as follows. Let $\langle x\rangle_{0}=G$. For $n \geq 1$, assume that $\langle x\rangle_{n-1}$ is defined. Then, $\langle x\rangle_{n}$ is the smallest normal subgroup of $\langle x\rangle_{n-1}$ containing $x$. The subgroup $\langle x\rangle_{n}$ is called the $n$-th normal closure (or the $n$-subnormal closure) of $x$ in $G$. Recall that a subnormal subgroup $N$ of $G$ is called $r$-subnormal in $G$ if

$$
r=\min \left\{r \in \mathbb{N} \mid \text { there exists a sequence } N=N_{r} \unlhd N_{r-1} \unlhd \ldots \unlhd N_{0}=G\right\} .
$$

It is easy to show that $\langle x\rangle_{n}$ is the intersection of all $s$-subnormal subgroups of $G$ containing $x$ with $s \leq n$ (see [13]). Before stating results on the subgroup $\langle x\rangle_{n}$, we borrow the following lemma.

Lemma 2.1 (Greenberg). Let $G$ be a free subgroup and $H$ a finitely generated subgroup of $G$. If $H$ contains a non-trivial subnormal subgroup of $G$, then $[G: H]<\infty$.

Proof. The lemma is implied by [3] or see [9, Theorem 2].

Lemma 2.2. Let $G$ be a free group of infinite rank. For a non-identity element $x$ of $G$ and a natural number $n$, the $n$-th normal closure $\langle x\rangle_{n}$ of $x$ in $G$ is free of infinite rank.

Proof. By the Nielsen-Schreier Theorem, every subgroup of a free group is free (see [15. pp. 103-104]), so $\langle x\rangle_{n}$ is free. By hypothesis, $\langle x\rangle_{0}=G$ is of infinite rank. Inductively, it suffices to show that $\langle x\rangle_{1}$ is of infinite rank. Assume that $\langle x\rangle_{1}$ is of finite rank. By Lemma 2.1, $\left[G:\langle x\rangle_{1}\right]<\infty$. Let $G^{\prime}$ be the commutator subgroup of $G$. Since $G$ is free of infinite rank, the abelian factor group $G / G^{\prime}$ is non-torsion and infinitely generated. Let $H=G^{\prime} \cdot\langle x\rangle_{1}$ be the subgroup of $G$ generated by $G^{\prime}$ and $\langle x\rangle_{1}$. Since $\langle x\rangle_{1}$ is of finite rank, $H / G^{\prime}$ is finitely generated. It follows that $G / H$ is infinitely generated, which contradicts the fact that $[G: H] \leq\left[G:\langle x\rangle_{1}\right]<\infty$. Thus, $\langle x\rangle_{1}$ is of infinite rank. The proof is complete.

Lemma 2.3. Let $G$ be a free group of infinite rank and $x$ an element of $G$. There exists a subgroup $H$ of $G$ satisfying the following conditions:

(1) $H$ is maximal in $G$.

(2) $H$ contains $x$. 
(3) $H$ is non-normal in $G$.

(4) $[G: H]$ is finite.

Proof. Let $G$ be generated by $\left\{x_{i} \mid i \in I\right\}$, where $I$ is an infinite set, and assume that $x=x_{i_{1}}^{n_{1}} x_{i_{2}}^{n_{2}} \ldots x_{i_{t}}^{n_{t}} \in G$ for some $i_{1}, \ldots, i_{t}$ in $I$ and for some integers $n_{1}, \ldots, n_{t}$. Denote by $S_{3}$ the symmetric group on $\{1,2,3\}$. Since $I$ is infinite, there exist some indices $\lambda, \mu \in I \backslash\left\{i_{1}, i_{2}, \ldots, i_{t}\right\}$ with $\lambda \neq \mu$. Let $\phi: G \rightarrow S_{3}$ be the group morphism defined by $x_{\lambda} \mapsto\left(\begin{array}{l}1 \\ 2\end{array}\right), x_{\mu} \mapsto\left(\begin{array}{ll}1 & 2\end{array} 3\right)$ and $x_{i} \mapsto$ Id for every $i \in I \backslash\{\lambda, \mu\}$. The group $N=\left\{\operatorname{Id},\left(\begin{array}{l}1 \\ 2\end{array}\right)\right\}$ is non-normal maximal subgroup of $S_{3}$ of index 3 . We show that the subgroup $H=\phi^{-1}(N)$ of $G$ satisfies the lemma. Observe that $\phi$ is onto, $H$ is non-normal in $G$ and $[G: H]=3$ implying $H$ is maximal in $G$. Finally, since $\phi(x)=\phi\left(x_{i_{1}}\right)^{n_{1}} \phi\left(x_{i_{2}}\right)^{n_{2}} \ldots \phi\left(x_{i_{t}}\right)^{n_{t}}=\operatorname{Id} \in N$, one has $x \in H$.

Next, we need to evaluate the depth of almost subnormal subgroups in free groups. In [13, A. Y. Olshanskii found a lower bound of depth for subnormal subgroups of $G$ contained in the $n$-th normal closure of a non-identity element. In fact, Olshanskii proved the following result.

Lemma 2.4. 13, Corollary 1.3] Let $G$ be a non-cyclic free group and $x$ be a nonidentity element of $G$. Assume that $n$ is a natural number and $H$ is a non-trivial subgroup of $\langle x\rangle_{n}$. If $H$ is s-subnormal in $G$, then $s \geq n$.

By using this lemma, we will show the following.

Lemma 2.5. Let $G$ be a free group of infinite rank, $x$ be a non-identity element of $G$ and $\langle x\rangle_{n}$ be the $n$-th normal closure of $x$ in $G$ for some positive integer $n$. If $\langle x\rangle_{n}$ contains a non-trivial subgroup $H$ which is almost s-subnormal in $G$, then $s \geq n$.

Proof. Assume that $H$ is a non-trivial subgroup of $\langle x\rangle_{n}$ such that $H$ is almost $s$-subnormal in $G$. Let

$$
H=H_{s}<H_{s-1}<\ldots<H_{1}<H_{0}=G
$$

be an almost normal series of $H$ in $G$, that is, for every $0 \leq i<s, H_{i+1}$ is normal in $H_{i}$ or $\left[H_{i}: H_{i+1}\right]$ is finite. We claim that $H$ contains a non-trivial subgroup $N$ which is $t$-subnormal in $G$ with $t \leq s$. We first observe that in the case when $\left[H_{s-1}: H\right]<\infty$, we denote by Core $(H)$ the core of $H$ in $H_{s-1}$. If Core $(H)$ is trivial, then since $\left[H_{s-1}: \operatorname{Core}(H)\right]<\infty$, one has $H_{s-1}$ is finite, so is $H$. Moreover, by the Nielsen-Schreier Theorem (see [15, pp. 103-104]), $H$ is a free group. It implies that $H$ is trivial, a contradiction. Hence, $\operatorname{Core}\left(H_{s}\right)$ is non-trivial. Therefore, by replacing $H$ by $\operatorname{Core}(H)$ if necessary, without loss of generality, we assume that $H=H_{s}$ is normal in $H_{s-1}$. We show the claim by induction on $s$. If $s=1$, then $H$ is normal in $G$, and the claim holds trivially. Assume that the claim is true for $s-1$, that is, every non-trivial almost $(s-1)$-subnormal subgroup of $G$ contains a non-trivial group which is $(t-1)$-subnormal in $G$ with $t \leq s$. In particular, $H_{s-1}$ contains a non-trivial subgroup $N_{s-1}$ which is $(t-1)$-subnormal in $G$. Put $N_{s}=H_{s} \cap N_{s-1}$. It is obvious that $N_{s}$ is normal in $N_{s-1}$. We consider two cases:

Case 1: $H_{s} \subseteq N_{s-1}$ or $N_{s-1} \subseteq H_{s}$.

It implies obviously that $N_{s}=H_{s} \cap N_{s-1}$ is non-trivial.

Case 2: $H_{s} \backslash N_{s-1} \neq \emptyset$ and $N_{s-1} \backslash H_{s} \neq \emptyset$. 
Let $h \in H_{s} \backslash N_{s-1}$ and $k \in N_{s-1} \backslash H_{s}$. Then, $h, k \in H_{s-1}$, so

$$
\left(h k h^{-1}\right) k^{-1}=h\left(k h^{-1} k^{-1}\right) \in H_{s} \cap N_{s-1}=N_{s} .
$$

If $h k h^{-1} k^{-1}=1$, then the subgroup $\langle h, k\rangle$ of $G$ is abelian. Again by the NielsenSchreier Theorem, $\langle h, k\rangle$ is a free group, which implies that $\langle h, k\rangle \cong \mathbb{Z}$. As a corollary, $h=k^{\alpha}$ for some $\alpha \in \mathbb{Z}$ or $k=h^{\beta}$ for some $\beta \in \mathbb{Z}$. This contradicts the assumption $h \in H_{s} \backslash N_{s-1}$ and $k \in N_{s-1} \backslash H_{s}$. Therefore, $N_{s}$ is non-trivial.

Both cases lead us to the conclusion that $N_{s}$ is non-trivial. It means that $N_{s}$, being a subgroup of $H_{s}$, is $t$-subnormal in $G$ with $t \leq s$. The claim is shown. Now, since $N_{s} \subseteq H_{s} \subseteq\langle x\rangle_{n}$, by Lemma 2.4 $t \geq n$. Thus, $s \geq t \geq n$. The proof is now complete.

Now, we fix some notation. Assume that $G$ is a free group generated by a countable set of indeterminates $\left\{x_{i} \mid i \in \mathbb{N} \backslash\{0\}\right\}$ with the Magnus order (see [10, Theorem 6.31] or see [11, Section 5.7] for further details). This order sometimes is called the dictionary order of free groups since it is induced from the dictionary order on $\left\{x_{i} \mid i \in \mathbb{N} \backslash\{0\}\right\}$. By reordering, we assume that $x_{1}<x_{2}<\ldots$ Observe that the order is a total order, that is, for every $a, b, c \in G$, the following conditions hold: (1) $a \leq b$ or $b \leq a$ (connex property); (2) if $a \leq b$ and $b \leq a$, then $a=b$ (antisymmetry); and (3) if $a \leq b$ and $b \leq c$, then $a \leq c$ (transitivity). Moreover, if $a \leq b$, then $a c \leq b c$ and $c a \leq c b$. Hence, $G$ is a total ordered group with dictionary order. Recall that a subset $S$ of $G$ is called well-ordered (briefly, WO) if every non-empty subset of $S$ has the least element. For a non-empty WO set $S$ of $G$, we denote by $\min (S)$ the least element of $S$.

Next, let $K$ be a division ring. From the general Mal'cev-Neumann construction of a Laurent series ring (we refer to [10, (14.21), p. 231] for details), we consider the Mal'cev-Neumann division ring $K((G, \Phi))$ of $G$ over $K$ with respect to a group homomorphism $\Phi: G \rightarrow \operatorname{Aut}(K), g \mapsto \Phi_{g}$.

More specifically, the Mal'cev-Neumann division ring $K((G, \Phi))$ consists of formal (but not necessarily finite) sums of the form $\alpha=\sum_{g \in G} a_{g} g$, where $a_{g} \in K$ and $\operatorname{supp}(\alpha)=\left\{g \in G \mid a_{g} \neq 0\right\}$ is WO. In $K((G, \Phi))$, addition and multiplication are defined for $\alpha=\sum_{g \in G} a_{g} g$ and $\beta=\sum_{g \in G} b_{g} g$ by:

$$
\alpha+\beta=\sum_{g \in G}\left(a_{g}+b_{g}\right) g
$$

and

$$
\alpha \beta=\sum_{t \in G}\left(\sum_{g h=t} a_{g} \Phi_{g}\left(b_{h}\right)\right) t .
$$

Now, we present the main result of this section by showing that the multiplicative group $K((G, \Phi))^{*}$ contains an almost subnormal subgroup of arbitrary depth $r$ which is not subnormal.

Theorem 2.6. Let $G$ be a free group generated by a countable set of indeterminates $\left\{x_{i}\right\}_{i \geq 1}$ with dictionary order, $K$ be a division ring and $\Phi: G \rightarrow \operatorname{Aut}(K)$ be a group morphism. Then, for every positive integer $r$, the multiplicative group $D^{*}$ of the Mal'cev-Neumann division ring $D=K((G, \Phi))$ contains an almost r-subnormal subgroup $N$ which is not subnormal in $D^{*}$. 
Proof. Let $x \in G \backslash\{1\}$ and consider the sequence of normal subgroups

$$
G=\langle x\rangle_{0} \triangleright\langle x\rangle_{1} \triangleright \ldots \triangleright\langle x\rangle_{r-1} .
$$

By lemmas 2.2 and 2.3. let $H$ be a maximal subgroup of $\langle x\rangle_{r-1}$ which is non-normal, of finite index in $\langle x\rangle_{r-1}$ and contains $x$. Then, in particular, $H$ is almost subnormal in $G$. Now put

$$
d: D^{*} \rightarrow G, \alpha \mapsto \min (\operatorname{supp}(\alpha))
$$

We claim that

$$
\min (\operatorname{supp}(\alpha \cdot \beta))=\min (\operatorname{supp}(\alpha)) \cdot \min (\operatorname{supp}(\beta)) \text { for every } \alpha, \beta \in D .
$$

Let $\alpha=\sum_{g \in G} a_{g} g, \beta=\sum_{g \in G} b_{g} g \in D$. Put

$$
A=\operatorname{supp}(\alpha), u=\min (A), B=\operatorname{supp}(\beta) \text { and } v=\min (B) .
$$

Then, $\alpha=\sum_{g \in A} a_{g} g$ and $\beta=\sum_{h \in B} b_{h} h$, where $a_{g} \neq 0$ and $b_{h} \neq 0$ for every $g \in A$ and $h \in B$. Observe that $u \leq g$ and $v \leq h$ for every $g \in A$ and $h \in B$, so $u v \leq t$ for every $t \in A$. B. Since $\alpha \beta=\sum_{t \in G} c_{t} t$, where $c_{t}=\sum_{g h=t, g \in A, h \in B} a_{g} \Phi_{g}\left(b_{h}\right)$, we have $u v \leq \min (\operatorname{supp}(\alpha \beta))$. Moreover, $c_{u v}=\sum_{g h=u v, g \in A, h \in B} a_{g} \Phi_{g}\left(b_{h}\right)$. One has $c_{u v}=$ $a_{u} \Phi_{u}\left(b_{v}\right) \neq 0$ because $u v \leq g h$ for every $g \in A$ and $h \in B$. So, $u v \in \operatorname{supp}(\alpha \beta)$. Therefore, $u v=\min (\operatorname{supp}(\alpha \beta))$, and the claim is shown. Consequently, $d$ is a group morphism and obviously $d$ is surjective. Put

$$
N=d^{-1}(H)=\{\alpha \in D \mid \min (\operatorname{supp}(\alpha)) \in H\} .
$$

We will show that $N$ is an almost $r$-subnormal subgroups of $D^{*}$ and it is not subnormal in $D^{*}$.

First, we show that $N$ is almost $r$-subnormal in $D^{*}$. Put

$$
N_{i}=d^{-1}\left(\langle x\rangle_{i}\right)=\left\{\alpha \in D \mid \min (\operatorname{supp}(\alpha)) \in\langle x\rangle_{i}\right\}
$$

for every $0 \leq i \leq r-1$. Then, $N<N_{r-1} \triangleleft N_{r-2} \triangleleft \ldots \triangleleft N_{0}=D^{*}$ with $\left[N_{r-1}: N\right]=$ $\left[\langle x\rangle_{r-1}: H\right]<\infty$ since $d$ is surjective. Therefore, $N$ is almost subnormal in $D^{*}$. Moreover, if

$$
N=X_{\ell}<X_{\ell-1}<\ldots<X_{1}<X_{0}=D^{*}
$$

is an almost normal series of $N$ in $D^{*}$, then

$$
H=d\left(X_{\ell}\right) \leq d\left(X_{\ell-1}\right) \leq \ldots \leq d\left(X_{1}\right) \leq d\left(X_{0}\right)=G
$$

is also an almost normal series of $H$ in $G$ since $d$ is surjective. According to Lemma 2.5, $\ell \geq r$, which implies that $N$ is almost $r$-subnormal in $D^{*}$.

Next, we claim that $N$ is not subnormal in $D^{*}$. Assume by contrary, $N$ is subnormal in $D^{*}$, and let

$$
N=N_{\ell} \unlhd N_{\ell-1} \unlhd \ldots \unlhd N_{1} \unlhd N_{0}=D^{*}
$$

be a normal series of $N$ in $D^{*}$. If $\ell<r$, then we add $N_{\ell+1}=N_{\ell+2}=\cdots=N_{r}$ to the normal series, so without loss of generality, we assume that $\ell \geq r$. Put $M_{i}=d\left(N_{i}\right)$ for every $0 \leq i \leq \ell$. Then, since $d$ is surjective,

$$
H=M_{\ell} \unlhd M_{\ell-1} \unlhd \ldots \unlhd M_{1} \unlhd M_{0}=G .
$$


By definition, $\langle x\rangle_{i}$ is contained in $M_{i}$ for every $0 \leq i \leq \ell$. Therefore, if $L_{i}=M_{i} \cap\langle x\rangle_{i}$ for every $1 \leq i \leq r$, then

$$
H=M_{\ell} \cap\langle x\rangle_{r} \unlhd M_{\ell-1} \cap\langle x\rangle_{r} \unlhd \ldots \triangleleft M_{r} \cap\langle x\rangle_{r}=\langle x\rangle_{r} .
$$

It implies that $H$ is subnormal in $\langle x\rangle_{r}$ which contradicts the fact that $H$ is maximal non-normal in $\langle x\rangle_{r}$. Thus, $N$ is non-subnormal in $D^{*}$, and the proof is now complete.

Using same arguments in the proof of Lemma 2.5. we can show that every nontrivial almost subnormal subgroup of a free group $G$ of infinite rank contains a non-trivial subnormal subgroup of $G$ and by repeating arguments in Theorem 2.6. we may show that if $D$ is the Mal'cev-Neumann division ring $K((G, \Phi))$ of a free group $G$ of infinite rank and $N$ is a non-central almost subnormal subgroup of $D^{*}$, then $N$ contains a non-central subnormal subgroup of $D^{*}$. It is natural to propose the following question.

Question 2.7. Let $D$ be a division ring and $N$ almost subnormal subgroup of $D^{*}$. If $N$ is non-central, then is it true that $N$ contains a non-central subnormal subgroup of $D^{*}$ ?

\section{3. $N$-INVARIANT DIVISION SUBRINGS}

The technique we use in this section is generalized rational identities. Now, let us recall the definition of generalized rational identities. Let $D$ be a division ring with center $F$ and $X=\left\{x_{1}, \ldots, x_{m}\right\}$ be a set of $m$ (noncommuting) indeterminates. We denote by $F\langle X\rangle$ the free $F$-algebra on $X$, by $D\langle X\rangle$ the free product of $D$ and $F\langle X\rangle$ over $F$, and by $D(X)$ the universal division ring of fractions of $D\langle X\rangle$. The existence of $D(X)$ was shown and studied deeply in [1, Chapter 7]. One calls an element $f(X) \in D(X)$ a generalized rational polynomial. If $f(X) \in D(X)$, then by [1, Theorem 7.1.2], $f(X)$ is an entry of the matrix $A^{-1}$, where $A \in M_{n}(D\langle X\rangle)$ for some positive integer $n$ such that $A$ is invertible in $M_{n}(D(X))$. Let $c=\left(c_{1}, \ldots, c_{m}\right) \in D^{m}$ and $\alpha_{c}: D\langle X\rangle \rightarrow D$ be the ring homomorphism defined by $\alpha\left(x_{i}\right)=c_{i}$. For any $n \in \mathbb{N}$, let $S(c, n)$ be the set of all square matrices $\left(f_{i j}(X)\right)$ of degree $n$ over $D\langle X\rangle$ such that the matrix $\left(f_{i j}(c)\right)$ is invertible in $M_{n}(D)$. Let $S(c)=\bigcup_{n \geq 1} S(c, n)$ and $E(c)$ be the subset of $D(X)$ consisting of all entries of $A^{-1}$, where $A$ ranges over $S(c)$. Then, $E(c)$ is a subring of $D(X)$ containing $D\langle X\rangle$ as a subring. Moreover, there is a ring homomorphism $\beta_{c}: E(c) \rightarrow D$ which extends $\alpha_{c}$ and every element of $E(c)$ is invertible if and only if the matrix mapped by $\beta_{c}$ is not zero. Let $f(X) \in D(X)$ and $c \in D^{m}$. If $f(X) \in E(c)$, then we say that $f(X)$ is defined at $c$ and $\beta_{c}(c)$ is denoted by $f(c)$. For any $f(X) \in D(X)$, the set of all $c \in D^{m}$ such that $f(X)$ is defined at $c$ is called the domain of $f(X)$ and is denoted by $\operatorname{Dom}_{D}(f)$. Let $S \subseteq D$ and $f(X)$ be a non-zero element in $D(X)$. If $f(c)=0$ for all $c \in S^{m} \cap \operatorname{Dom}_{D}(f)$, then we say that $f=0$ is a generalized rational identity of $S$ or $S$ satisfies the generalized rational identity $f=0$. In this paper, we borrow the following result.

Lemma 3.1. [4, Theorem 1.1] Let $D$ be a division ring with infinite center. If $D^{*}$ contains a non-central almost subnormal subgroup satisfying a generalized rational identity, then $D$ is centrally finite, that is, $D$ is finite-dimensional over its center. 
In this section, we mainly work with a special class of generalized rational identities, namely, generalized group identities. An element

$$
w\left(x_{1}, x_{2}, \ldots, x_{n}\right)=a_{1} x_{i_{1}}^{m_{1}} a_{2} x_{i_{2}}^{m_{2}} \ldots a_{t} x_{i_{t}}^{m_{t}} a_{t+1} \in D(X)
$$

is called a generalized group monomial over $D^{*}$ if $a_{1}, a_{2}, \ldots, a_{t+1} \in D^{*}$. Additionally, assume that $w$ is non-identity. Let $H$ be a subgroup of $D^{*}$. We say that $w=1$ is a generalized group identity of $H$ or $H$ satisfies the generalized group identity $w=1$ if $w\left(c_{1}, c_{2}, \ldots, c_{n}\right)=1$ for every $c_{1}, c_{2}, \ldots, c_{n} \in H$. For results on generalized group identities in division rings, we prefer [2] and [17. Results on generalized group identities of almost subnormal subgroups in division rings we use in this paper are from 12 . In fact, we will use the following result.

Proposition 3.2. [12, Theorem 2.2] Let $D$ be a division ring with infinite center $F$ and assume that $N$ is an almost subnormal subgroup of $D^{*}$. If $N$ satisfies a generalized group identity over $D^{*}$, then $N$ is central, that is, $N \subseteq F$.

Assume that $H$ is a subgroup of finite index $n$ in a group $G$. If $H$ is normal in $G$, then $g^{n} \in H$ for any element $g \in G$. If $H$ is non-normal in $G$, then by Poincare's theorem, it is easy to see that $g^{n !} \in H$ for any element $g \in G$.

Let $a \in D^{*}$. Assume that $N$ is a non-central almost subnormal subgroup of $D^{*}$ with an almost normal series

$$
N=N_{r} \leq N_{r-1} \leq \ldots \leq N_{1} \leq N_{0}=D^{*} .
$$

We construct a series of subgroups $H_{n}$ of $D^{*}$ depending on $N$ and $a$ as follows:

Put $H_{0}=D^{*}$. For any integer $n>0$, if $N_{n}$ is normal in $N_{n-1}$, then we put $H_{n}:=\left\langle b a b^{-1} \mid b \in H_{n-1}\right\rangle$. Otherwise, $\left[N_{n-1}: N_{n}\right]=\ell_{n}<\infty$, we put $H_{n}:=\left\langle b^{\ell_{n}} ! \mid b \in H_{n-1}\right\rangle$. Hence, we get the following new sequence of subgroups

$$
H=H_{r} \leq H_{r-1} \leq \ldots \leq H_{1} \leq H_{0}=D^{*} .
$$

Moreover, we have the following lemma.

Lemma 3.3. Let $N_{1}, N_{2}, \ldots, N_{r}, H_{1}, H_{2}, \ldots, H_{r}$ and $a$ be as above. Then, the following statements hold:

(1) For every $0 \leq n<r, H_{n} \unlhd H_{n-1}$ in case $N_{n} \unlhd N_{n-1}$, and $b^{\ell_{n}} \in H_{n}$ for every $b \in H_{n-1}$ in case $\left[N_{n}: N_{n-1}\right]=\ell_{n}<\infty$.

(2) If $a \in N$, then $H_{n} \leq N_{n}$ for any $0 \leq n \leq r$.

(3) For any $0 \leq n \leq r$, if $c \in D^{*}$ such that ac $=c a$, then $c H_{n} c^{-1} \leq H_{n}$.

Proof. (1) It follows immediately from the definition of $H_{n}$.

(2) We show this assertion by induction on $n$. It is trivial that $H_{0} \subseteq N_{0}$. Assume that $H_{n-1} \leq N_{n-1}$. We will show $H_{n} \leq N_{n}$. There are two cases.

Case 1: $N_{n} \unlhd N_{n-1}$.

Since $a \in N \leq N_{n}$, one has $b a b^{-1} \in N_{n}$ for every $b \in N_{n-1}$. In particular, $b a b^{-1} \in N_{n}$ for every $b \in H_{n-1}$. Hence, $H_{n}:=\left\langle b a b^{-1} \mid b \in H_{n-1}\right\rangle \leq N_{n}$.

Case 2. $\left[N_{n-1}: N_{n}\right]=\ell_{n}<\infty$.

Then, $b^{\ell_{n} !} \in N_{n}$ for every $b \in N_{n-1}$. In particular, $b^{\ell_{n} !} \in N_{n}$ for every $b \in H_{n-1}$. Therefore,

$$
H_{n}:=\left\langle b^{\ell_{n} !} \mid b \in H_{n-1}\right\rangle \leq N_{n}
$$


In both cases, we have $H_{n} \leq N_{n}$, so (2) holds.

(3) We prove this statement by induction on $n$. It is trivial that $c H_{0} c^{-1}=H_{0}$. Assume that $0<n \leq r$ and $c H_{n-1} c^{-1} \leq H_{n-1}$. We must show $c H_{n} c^{-1} \leq H_{n}$. If $N_{n}$ is normal in $N_{n-1}$, then $H_{n}=\left\langle b a b^{-1} \mid b \in H_{n-1}\right\rangle$. Hence,

$$
c H_{n} c^{-1}=\left\langle c\left(b a b^{-1}\right) c^{-1} \mid b \in H_{n-1}\right\rangle=\left\langle\left(c b c^{-1}\right) a\left(c b c^{-1}\right)^{-1} \mid b \in H_{n-1}\right\rangle \leq H_{n} .
$$

In the case when $\left[N_{n-1}: N_{n}\right]=\ell_{n}<\infty$, then $b^{\ell_{n} !} \in N_{n}$ for any $b \in N_{n-1}$. By definition, $H_{n}=\left\langle b^{\ell_{n}} ! \mid b \in H_{n-1}\right\rangle$, so

$$
c H_{n} c^{-1}=\left\langle c b^{\ell_{n} !} c^{-1} \mid b \in H_{n-1}\right\rangle=\left\langle\left(c b c^{-1}\right)^{\ell_{n} !} \mid b \in H_{n-1}\right\rangle \leq H_{n} .
$$

The proof is now complete.

Now, keeping the assumption and the notation for $N$ as above, we build inductively a sequence of non-identity elements $w_{n}$ in $D(x, y)$ depending on $N$ as follows. Put $w_{0}(x, y)=x$. For any integer $n \geq 1$, put

$$
w_{n}(x, y):= \begin{cases}{\left[w_{n-1}(x, y), y\right],} & \text { if } N_{n} \text { is normal in } N_{n-1} \text { or } n>r \\ \left(w_{n-1}(x, y)\right)^{\ell_{n} !}, & \text { if }\left[N_{n-1}: N_{n}\right]=\ell_{n}<\infty .\end{cases}
$$

We claim that if $a \in D^{*}$ and $b \in N$, then $w_{n}(a, b) \in N_{n}$ for any $n \leq r$ and $w_{n}(a, b) \in N_{r}=N$ for any $n>r$. We will first show the claim by induction on $0 \leq$ $n \leq r$. It is clear that $w_{0}(a, b)=a \in N_{0}=D^{*}$. Assume that $w_{n-1}(a, b) \in N_{n-1}$. If $N_{n}$ is normal in $N_{n-1}$, then

$$
w_{n}(a, b)=w_{n-1}(a, b) b\left(w_{n-1}(a, b)\right)^{-1} b^{-1}=\left(w_{n-1}(a, b) b\left(w_{n-1}(a, b)\right)^{-1}\right) b^{-1} \in N_{n} .
$$

If $\left[N_{n-1}: N_{n}\right]=\ell_{n}<\infty$, then $c^{\ell_{n} !} \in N_{n}$ for every $c \in N_{n-1}$, so $w_{n}(a, b)=$ $\left(w_{n-1}(a, b)\right)^{\ell_{n} !} \in N_{n}$. Thus, we showed that $w_{n}(a, b) \in N_{n}$ for any $n \leq r$. As a corollary, $w_{n}(a, b) \in N_{r}=N$ for any $n>r$.

Now, assume that $K$ is a division subring of $D$ such that $K$ is $N$-invariant and $K \nsubseteq F$. Suppose that $h \in(K \cap N) \backslash F$ and $g \in N \backslash\{-1\}$, and consider the elements

$$
\begin{aligned}
& u_{n}(h, g):=w_{n}\left((1+g) h(1+g)^{-1}, g\right), \\
& v_{n}(h, g):=w_{n}\left((1+g)^{-1} h(1+g), g\right) .
\end{aligned}
$$

Lemma 3.4. For any $n \geq 0, u_{n}(h, g)=(1+g) \phi_{n}(h, g)(1+g)^{-1}$ and $v_{n}(h, g)=$ $(1+g)^{-1} \phi_{n}(h, g)(1+g)$, where $\phi_{n}(h, g)$ is an element of the subgroup $\langle h, g\rangle$ of $K^{*} \cap N$.

Proof. We prove the lemma by induction on $n$. We have

$$
u_{0}(h, g)=w_{0}\left((1+g) h(1+g)^{-1}, g\right)=(1+g) h(1+g)^{-1}
$$

and

$$
v_{0}(h, g)=w_{0}\left((1+g)^{-1} h(1+g), g\right)=(1+g)^{-1} h(1+g) .
$$

Hence, $\phi_{0}(h, g)=h \in\langle h, g\rangle$.

Assume that $u_{n-1}=w_{n-1}\left((1+g) h(1+g)^{-1}, g\right)=(1+g) \phi_{n-1}(h, g)(1+g)^{-1}$ and $v_{n-1}=w_{n-1}\left((1+g)^{-1} h(1+g), g\right)=(1+g)^{-1} \phi_{n-1}(h, g)(1+g)$ where $\phi_{n-1}(h, g)$ is an element of the subgroup $\langle h, g\rangle$. Now consider $u_{n}=w_{n}\left((1+g) h(1+g)^{-1}, g\right)$ and $v_{n}=w_{n}\left((1+g)^{-1} h(1+g), g\right)$ with two cases:

Case 1: $N_{n}$ is normal in $N_{n-1}$. 
Then,

$$
\begin{aligned}
u_{n}(h, g) & =w_{n}\left((1+g) h(1+g)^{-1}, g\right) \\
& =w_{n-1}\left((1+g) h(1+g)^{-1}, g\right) g\left(w_{n-1}\left((1+g) h(1+g)^{-1}, g\right)\right)^{-1} g^{-1} \\
& =(1+g) \phi_{n-1}(h, g)(1+g)^{-1} g(1+g)\left(\phi_{n-1}(h, g)\right)^{-1}(1+g)^{-1} \\
& =(1+g) \phi_{n-1}(h, g) g\left(\phi_{n-1}(h, g)\right)^{-1}(1+g)^{-1} .
\end{aligned}
$$

It is similar that

$$
v_{n}(h, g)=(1+g)^{-1} \phi_{n-1}(h, g) g\left(\phi_{n-1}(h, g)\right)^{-1}(1+g) .
$$

It implies that $\phi_{n}(h, g)=\phi_{n-1}(h, g) g\left(\phi_{n-1}(h, g)\right)^{-1} \in\langle h, g\rangle$.

Case 2: $\left[N_{n-1}: N_{n}\right]=\ell_{n}<\infty$.

Then,

$$
u_{n}(h, g)=w_{n}\left((1+g) h(1+g)^{-1}, g\right)^{\ell_{n} !}=(1+g)\left(\phi_{n-1}(h, g)\right)^{\ell_{n} !}(1+g)^{-1} .
$$

Similarly, $v_{n}(h, g)=(1+g)^{-1}\left(\phi_{n-1}(h, g)\right)^{\ell_{n} !}(1+g)$. Hence,

$$
\phi_{n}(h, g)=\left(\phi_{n-1}(h, g)\right)^{\ell_{n} !} \in\langle h, g\rangle .
$$

The proof is now complete.

Lemma 3.5. Let $D$ be a division ring with infinite center $F$, and assume that $N$ is a non-central almost subnormal subgroup of $D^{*}$. If $K$ is a non-central $N$-invariant division subring of $D$, then $K \cap N$ is non-abelian. In particular, $K \cap N \nsubseteq F$.

Proof. We will show that $K^{*} \cap N$ is not abelian. Assume that $K^{*} \cap N$ is abelian, and take any $a \in K \backslash F$. For any $b \in N$, we claim that $w_{r}(a, b) \in K^{*} \cap N$. As we have noted above, $w_{r}(a, b) \in N_{r}=N$. Now, we prove by induction that $w_{n}(a, b) \in K^{*}$ on $n \geq 0$. In the beginning, we have $w_{0}(a, b)=a \in K^{*}$. Suppose that $w_{n-1}(a, b) \in K^{*}$, then consider $w_{n}(a, b)$. If $N_{n}$ is normal in $N_{n-1}$, then $w_{n}(a, b)=\left[w_{n-1}(a, b), b\right]=w_{n-1}(a, b) b\left(w_{n-1}(a, b)\right)^{-1} b^{-1}$. It implies that $w_{n+1}(a, b) \in K^{*}$ because $b\left(w_{n-1}(a, b)\right)^{-1} b^{-1} \in K^{*}$ in view of the assumption that $K$ is $N$-invariant. If $\left[N_{n-1}: N_{n}\right]=\ell_{n}<\infty$, then $w_{n}(a, b)=\left(w_{n-1}(a, b)\right)^{\ell_{n} !} \in K^{*}$. By the inductive assumption, $w_{n}(a, b) \in K^{*}$ for any $n \geq 0$. In particular, $w_{r}(a, b) \in$ $K^{*} \cap N$, and the claim is proved. Thus, $w_{r}(a, b) w_{r}(c, d)\left(w_{r}(a, b)\right)^{-1}\left(w_{r}(c, d)\right)^{-1}=1$ for any $a, c \in K \backslash F$ and $b, d \in N$ because $K^{*} \cap N$ is abelian. Since $a, c \notin F$, $w_{r}(a, x) w_{r}(c, y)\left(w_{r}(a, x)\right)^{-1}\left(w_{r}(c, y)\right)^{-1}$ is non-identity in $D(x, y)$. Hence, $N$ satisfies the generalized group identity

$$
w_{r}(a, x) w_{r}(c, y)\left(w_{r}(a, x)\right)^{-1}\left(w_{r}(c, y)\right)^{-1}=1 .
$$

By Proposition 3.2. $N$ is central, a contradiction.

The following lemma is a special case of Theorem 3.10 when $K$ is assumed to be a subfield of $D$.

Lemma 3.6. Let $D$ be a division ring with infinite center $F$, and assume that $N$ is a non-central almost subnormal subgroup of $D^{*}$. If $K$ is an $N$-invariant subfield of $D$, then $K \subseteq F$. 
Proof. Assume that $K$ is a subfield of $D$ which is non-central and $N$-invariant. Let $a \in K \backslash F$. Then, for every $b \in N$, one has $b a b^{-1} \in K$, so $b a b^{-1} a b a^{-1} b^{-1} a^{-1}=$ $\left(b a b^{-1}\right) a\left(b a b^{-1}\right)^{-1} a^{-1}=1$. Moreover, $x a x^{-1} a x a^{-1} x^{-1} a^{-1}$ is non-identity in $D(x)$ as $a \notin F$. Hence, $N$ satisfies the generalized group identity

$$
x a x^{-1} a x a^{-1} x^{-1} a^{-1}=1 .
$$

Now, by Proposition [3.2, $N$ is central and this contradicts the hypothesis. The proof is now complete.

The following lemma is essential in the proof of Theorem 3.10 .

Lemma 3.7. Let $D$ be a division ring with infinite center $F$, and assume that $N$ is a non-central almost subnormal subgroup of $D^{*}$. If $K$ is a non-central $N$-invariant division subring of $D$, then $C_{D}(a) \subseteq K$ for any $a \in(K \cap N) \backslash F$.

Proof. Since $K \nsubseteq F$ by Lemma 3.5, we have $K \cap N \nsubseteq F$. Assume that there exists $a \in(K \cap N) \backslash F$ such that $C_{D}(a) \nsubseteq K$. Let

$$
N=N_{r} \leq N_{r-1} \leq \ldots \leq N_{1} \leq N_{0}=D^{*}
$$

be an almost normal series of $N$ in $D^{*}$.

Consider the subgroups $H_{n}$ constructed as in Lemma 3.3 for $0 \leq n \leq r$, that is, $H_{0}=D^{*}$. For any integer $n>0$, if $N_{n}$ is normal in $N_{n-1}$, then we put $H_{n}:=\left\langle b a b^{-1} \mid b \in H_{n-1}\right\rangle$. Otherwise, that is, $\left[N_{n-1}: N_{n}\right]=\ell_{n}<\infty$, we put $H_{n}:=\left\langle b^{\ell_{n}} ! \mid b \in H_{n-1}\right\rangle$. Let $g \in H_{r}$ be arbitrary. Since $H_{r} \leq N_{r}=N$, $\operatorname{gag}^{-1} \in K^{*}$. We first claim that

$$
C_{D}(a)=C_{D}\left(g a g^{-1}\right)=g C_{D}(a) g^{-1} .
$$

For $b \in C_{D}(a)$, put

$$
h:=b\left(g a g^{-1}\right) b^{-1}=\left(b g b^{-1}\right) a\left(b g b^{-1}\right)^{-1} .
$$

By Lemma 3.3, $b g b^{-1} \in b H_{r} b^{-1} \leq H_{r} \leq N_{r} \leq N$. Since $K$ is $N$-invariant, $h \in K$ and $b\left(g a g^{-1}\right)=h b$. Similarly, since $b+1 \in C_{D}(a),(b+1)\left(g a g^{-1}\right)=h^{\prime}(b+1)$ for some $h^{\prime} \in K$. Hence,

$$
g a g^{-1}=(b+1)\left(g a g^{-1}\right)-b\left(g a g^{-1}\right)=\left(h^{\prime}-h\right) b+h^{\prime} .
$$

Recall that $\mathrm{gag}^{-1} \in K$, so, if $h^{\prime} \neq h$ then

$$
b=\left(h^{\prime}-h\right)^{-1}\left(g a g^{-1}-h^{\prime}\right) \in K .
$$

Therefore, if $b \in C_{D}(a) \backslash K$, then $h=h^{\prime}$, equivalently,

$$
b\left(g_{a g}^{-1}\right)=h b=h^{\prime} b=\left(g_{a g}{ }^{-1}\right) b .
$$

Hence, $b \in C_{D}\left(g_{a g}^{-1}\right)$ for $b \in C_{D}(a) \backslash K$. As a result, $C_{D}(a) \backslash K \subseteq C_{D}\left(g^{2} g^{-1}\right)$. Now, for any $b^{\prime} \in K \cap C_{D}(a)$, one has $b+b^{\prime} \in C_{D}(a) \backslash K \subseteq C_{D}\left(g_{a g}^{-1}\right)$, so $(b+$ $\left.b^{\prime}\right)\left(g a g^{-1}\right)=\left(g a g^{-1}\right)\left(b+b^{\prime}\right)$, equivalently, $b^{\prime}\left(g a g^{-1}\right)=\left(g a g^{-1}\right) b^{\prime}$. Thus,

$$
C_{D}(a) \cap K \subseteq C_{D}\left(g_{a g}^{-1}\right) .
$$

As a corollary, $C_{D}(a) \subseteq C_{D}\left(g a g^{-1}\right)$. Replacing $a$ by $g a g^{-1} \in K \cap N$ and $g$ by $g^{-1}$, we have $C_{D}\left(g a g^{-1}\right) \subseteq C_{D}\left(g^{-1}\left(g a g^{-1}\right) g\right)=C_{D}(a)$. Hence, $C_{D}(a)=C_{D}\left(g a g^{-1}\right)$. Now, we prove the equality $C_{D}(a)=g C_{D}(a) g^{-1}$. For any $b \in C_{D}(a)=C_{D}\left(g_{a g}^{-1}\right)$, one has

$$
\left(g b g^{-1}\right) a=g b\left(g^{-1} a g\right) g^{-1}=g\left(g^{-1} a g\right) b g^{-1}=a\left(g b g^{-1}\right),
$$


so $g b g^{-1} \in C_{D}(a)$. Therefore, the inclusion $g C_{D}(a) g^{-1} \subseteq C_{D}(a)$ holds for an arbitrary element $g \in H_{r}$. So, it follows $g^{-1} C_{D}(a) g \subseteq C_{D}(a)$, and consequently we have $C_{D}(a)=g C_{D}(a) g^{-1}$. The claim is now proved.

Now, we claim that the center $Z\left(C_{D}(a)\right)$ of $C_{D}(a)$ is $H_{r}$-invariant. For any $g \in H_{r}, c \in Z\left(C_{D}(a)\right)$ and $d \in C_{D}(a)$, we have $\left(g c g^{-1}\right) d=g c\left(g^{-1} d g\right) g^{-1}=$ $g\left(g^{-1} d g\right) c g^{-1}=d\left(g c g^{-1}\right)$. By applying Lemma 3.6 for the subgroup $H_{r}$ and subfield $Z\left(C_{D}(a)\right)$, we have $Z\left(C_{D}(a)\right) \subseteq F$. In particular, $a \in F$, a contradiction. Thus, the proof is now complete.

Lemma 3.8. [14, 14.3.6, p. 435] Let $K$ be a division subring of $D$. If $g \in D \backslash K$ such that $g K g^{-1} \subseteq K$, then $K \cap(1+g) K(1+g)^{-1}=C_{D}(g) \cap K$.

Corollary 3.9. Let $D$ be a division ring with infinite center $F$, and assume that $N$ is a non-central almost subnormal subgroup of $D^{*}$. Assume that $K$ is an $N$ invariant division subring of $D$. If there exists $g \in N \backslash K$, then

$$
N \cap K \cap(1+g) K(1+g)^{-1} \subseteq F .
$$

Proof. Assume that $N \cap K \cap(1+g) K(1+g)^{-1} \subseteq F$ for every $g \in N \backslash K$. By Lemma 3.8, there exists $h \in\left(N \cap K \cap C_{D}(g)\right) \backslash F$. Hence, $g \in C_{D}(h)$, but by Lemma 3.7. $C_{D}(h) \subseteq K$, so $g \in K$ which contradicts the assumption.

Now, we are ready to prove the main result in this paper.

Theorem 3.10. Let $D$ be a division ring with infinite center $F$ and $K$ be a division subring of $D$. Assume that $N$ is a non-central almost subnormal subgroup of $D^{*}$. If $K$ is $N$-invariant, then $K \subseteq F$ or $K=D$.

Proof. Assume that $N$ is an almost $r$-subnormal subgroup in $D^{*}$ with an almost normal series

$$
N=N_{r} \leq N_{r-1} \leq \ldots \leq N_{0}=D^{*} .
$$

Observe that if $N_{r}$ has finite index in $N_{r-1}$, then the core

$$
\text { Core }_{N_{r-1}}\left(N_{r}\right):=\bigcap_{x \in N_{r-1}} N_{r}^{x}
$$

of $N_{r}$ in $N_{r-1}$ is a normal subgroup of finite index in $N_{r-1}$.

We claim that Core $_{N_{r-1}}\left(N_{r}\right)$ is non-central. Indeed, if $\operatorname{Core}_{N_{r-1}}\left(N_{r}\right)$ is central, then $a^{n} \in \operatorname{Core}_{N_{r-1}}\left(N_{r}\right)$ for any $a \in N_{r-1}$, where $n=\left[N_{r-1}: \operatorname{Core}_{N_{r-1}}\left(N_{r}\right)\right]$. Hence, $a^{n} b^{n} a^{-n} b^{-n}=1$ for very $a, b \in N_{r-1}$. Moreover, $x^{n} y^{n} x^{-n} y^{-n}$ is nonidentity, so $N_{r-1}$ satisfies the identity $x^{n} y^{n} x^{-n} y^{-n}=1$. By [12, Theorem 2.2], $N_{r-1}$ is central, so is $N=N_{r} \subseteq F$, a contradiction. To prove the theorem, it suffices to use the fact that $K$ is normalized by the core of $N_{r}$ in $N_{r-1}$. So, without loss of generality, we can assume that $N_{r}$ is normal in $N_{r-1}$. We shall prove the theorem firstly for the case when $K$ is centrally finite, that is, when $K$ is a finite dimensional vector space over its center $Z(K)$, and then for the general case.

Case $1 . K$ is centrally finite.

We shall prove the statement by induction on $r$. If $r=0$, then $K^{*}$ is normal in $D^{*}$, and the statement is true by the Carter-Brauer-Hua Theorem. Assume that the statement holds for any almost subnormal subgroup in $D^{*}$ of depth $<r$. Assuming $K \nsubseteq F$, we must show $K=D$. In view of Lemma 3.6. $K$ is not commutative. We 
claim that $Z(K)$ is contained in $F$. Indeed, for any $x \in Z(K), g \in N_{r}$ and $h \in K$, since $g K g^{-1}=K$, there exists $h^{\prime} \in K$ such that $h=g h^{\prime} g^{-1}$. One has

$$
\left(g x g^{-1}\right) h=g x g^{-1} g h^{\prime} g^{-1}=g x h^{\prime} g^{-1}=g h^{\prime} x g^{-1}=g h^{\prime} g^{-1} g x g^{-1}=h\left(g x g^{-1}\right) .
$$

Hence, $g x g^{-1} \in Z(K)$ for any $x \in Z(K)$ and $g \in N_{r}$. This means that $Z(K)$ is $N_{r}$-invariant. By Lemma 3.6. $Z(K) \subseteq F$. The claim is proved. Since $K \nsubseteq F$, by Lemma 3.5. there exists an element $h \in(K \cap N) \backslash F$. Assume that $N_{r} \nsubseteq \mathbb{K}$. Then, there exists $g \in N_{r} \backslash K$. Consider the morphism $\psi: K \rightarrow K$ defined by $\psi(x)=g x g^{-1}$ for any $x \in K$. This morphism is a $Z(K)$-automorphism of $K$ in view of the inclusion $Z(K) \subseteq F$. Since $K$ is finite dimensional over $Z(K)$, by the Skolem-Noether Theorem, there exists $a \in K^{*}$ such that $\psi(x)=a x a^{-1}$ for any $x \in K$. In particular, $g h g^{-1}=a h a^{-1}$, equivalently, $\left(a^{-1} g\right) h=h\left(a^{-1} g\right)$. Hence, $a^{-1} g \in C_{D}(h)$, but in view of Lemma 3.7. $C_{D}(h) \subseteq K$, so we have $g \in K$, a contradiction. Thus, $N_{r} \subseteq K$. As a corollary, the division subring $L$ of $D$ generated by $N_{r}$ is contained in $K$ and $L$ is $N_{r-1}$-invariant. By the inductive, $L=D$, and consequently, $K=D$.

Case 2. General case.

As in Case 1, we shall prove the statement by induction on $r$. If $r=0$, then the statement is true by the Carter-Brauer-Hua Theorem. Assume that the statement holds for any almost subnormal subgroup of $D$ of depth $<r$. Assuming $K \nsubseteq F$, we must show $K=D$. We claim that $N_{r} \subseteq K$. Assume that $N_{r} \nsubseteq K$. Then, take $g \in N_{r} \backslash K$, and by Lemma 3.5. let $h \in\left(K \cap N_{r}\right) \backslash F$. Consider $u=u_{r}(h, g)=$ $(1+g) \phi_{n}(h, g)(1+g)^{-1} \in N_{r}$ and $v=v_{r}(h, g)=(1+g)^{-1} \phi_{n}(h, g)(1+g) \in N_{r}$ as in Lemma 3.4. One has

$$
\begin{aligned}
\alpha(h, g) & =\left[\phi_{n}(h, g), u\right]=\left[(1+g) v(1+g)^{-1},(1+g) \phi_{n}(h, g)(1+g)^{-1}\right] \\
& =(1+g)\left[v, \phi_{n}(h, g)\right](1+g)^{-1} \in(1+g) K(1+g)^{-1} .
\end{aligned}
$$

On the other hand $\alpha(h, g)=\left[\phi_{n}(h, g), u\right] \in\left[K \cap N_{r}, N_{r}\right] \subseteq K \cap N_{r}$. Hence, $\alpha(h, g) \in$ $N_{r} \cap K \cap(1+g) K(1+g)^{-1}$. In view of Corollary 3.9, $\alpha(h, g) \in F$. Observe that for any $g^{\prime} \in K \cap N_{r}, g g^{\prime} \in N_{r} \backslash K$, so $\alpha\left(h, g g^{\prime}\right) \in F \cap K$. Let us fix some element $e \in\left(K \cap N_{r}\right) \backslash F$, and recall elements $w_{n}(a, b)$ which have been constructed before Lemma 3.4 for $a, b \in D^{*}$ and $n \geq 0$. We claim that $\alpha\left(h, g w_{r+1}(d, e)\right) \in F \cap K \subseteq$ $Z(K)$ for any $d \in D^{*}$. To do this, it suffices to show that $w_{r+1}(d, e) \in N_{r} \cap K$ for any $d \in D^{*}$. Recall that $e \in N_{r} \cap K$, so by a remark before Lemma 3.4, we have $w_{r}(d, e) \in N_{r}$ for any $d \in D^{*}$. Moreover, since $K$ is $N_{r}$-invariant, we also have

$$
w_{r+1}(d, e)=\left[w_{r}(d, e), e\right]=w_{r}(d, e) e\left(w_{r}(d, e)\right)^{-1} e^{-1} \in K \cap N_{r} .
$$

Thus, we have shown that $\alpha\left(h, g w_{r}(p, e)\right) \in F$ for every $c \in D^{*}$. Hence,

$$
\alpha\left(h, g w_{r}(p, e)\right) q-q \alpha\left(h, g w_{r}(p, e)\right)=0
$$

for every $p, q \in D^{*}$. Since $\alpha\left(h, g w_{r}(x, e)\right) y-y \alpha\left(h, g w_{r}(x, e)\right)$ is non-zero in $D(x, y)$, $D^{*}$ satisfies generalized rational identity $\alpha\left(h, g w_{r}(x, e)\right) y-y \alpha\left(h, g w_{r}(x, e)\right)=0$. Therefore, by Lemma 3.1, $D$ is centrally finite, so in view of [5, Theorem 3], $K$ is centrally finite. By Case $1, K=D$. But this fact contradicts the assumption that $N_{r} \nsubseteq K$. Thus, $N_{r} \subseteq K$, and the claim is proved. Now, as in Case 1, we conclude that the division subring $L$ of $D$ generated by $N_{r}$ is $N_{r-1}$-invariant, so $L=D$ by the inductive, and this implies $K=D$. The proof of the theorem is now complete. 


\section{REFERENCES}

[1] P. M. Cohn, Free rings and their relations, Academic Press, New York and London, 1971.

[2] I. Z. Golubchik and A. V. Mikhalev, Generalized group identities in the classical groups, Zap. Nauch. Semin. LOMI AN SSSR 114 (1982): 96-119.

[3] L. Greenberg, Discrete groups of motions, Canad. J. Math. 12 (1960), 414-425.

[4] B. X. Hai, M. H. Bien and T. H. Dung, Generalized algebraic rational identities of subnormal subgroups in division rings, arXiv:1709.04774v1[math RA]13 Sep. 2017.

[5] B. X. Hai and N. K. Ngoc, A note on the existence of non-cyclic free subgroups in division rings, Archiv der Math. 101 (2013), 437-443.

[6] B. Hartley, Free groups in normal subgroups of unit groups and arithmetic groups, Contemp. Math. 93 (1989) 173-177.

[7] R. Hazrat and A.R. Wadsworth, On maximal subgroups of the multiplicative group of a division algebra, J. Algebra 322 (2009), 2528-2543.

[8] I. N. Herstein and W. R. Scott, Subnormal subgroups of division rings, Canad. J. Math. 15 (1963) 80-83.

[9] A. Karrass and D. Solitar, On Finitely Generated Subgroups of a Free Group, Proc. Amer. Math. Soc. 22 (1969), 209-213.

[10] T. Y. Lam, A first course in noncommutative Rings, GMT 131, Springer, 1991.

[11] W. Magnus, A. Karrass and D. Solitar, Combinatorial group theory. Presentation of groups in terms of generators and relations. 2nd revised edition, Dover Publications, New York, 1976.

[12] N. K. Ngoc, M. H. Bien and B. X. Hai, Free subgroups in almost subnormal subgroups of general skew linear groups, St. Petersburg Math. J. 28 (2017), 707-717.

[13] A. Y. Olshanskii, Subnormal subgroups in free groups, their growth and cogrowth, Math. Proc. Camb. Phil. Soc. 169 (2017), 499-531.

[14] W. R. Scott, Group theory, Dover Publications Inc., New York, second edition, 1987.

[15] J. Stillwell, Classical Topology and Combinatorial Group Theory, In: Graduate Texts in Mathematics, Vol 72, 2nd edition, Springer-Verlag, 1993.

[16] C. J. Stuth, A generalization of the Cartan-Brauer-Hua Theorem, Proc. Amer. Math. Soc. 15 (1964), 211-217.

[17] G. M. Tomanov, Generalized group identities in linear groups, Math. USSR, Sbornik 51 (1985), 33-46.

[18] B.A. F. Wehrfritz, A note on almost subnormal subgroups of linear groups, Proc. Amer. Math. Soc. 117 (1993), no. 1, 17-21.

E-mail address: ttdeo@hcmus.edu.vn; mhbien@hcmus.edu.vn, bxhai@hcmus.edu.vn

Faculty of Mathematics and Computer Science, VnuhCm - University of Science, 227 Nguyen Van Cu Str., Dist. 5, Ho Chi Minh City, Vietnam. 